\title{
Stress and Resilience for Parents of Children With Intellectual and Developmental Disabilities: A Review of Key Factors and Recommendations for Practitioners
}

Justin W. Peer and Stephen B. Hillman ${ }^{\dagger}$

${ }^{\star}$ Behavioral Sciences Department, University of Michigan-Dearborn, Dearborn, MI, USA; and ${ }^{\dagger}$ College of Education, Wayne State University, Detroit, MI, USA

\begin{abstract}
Parents of children with intellectual and/or developmental disabilities experience more stress in comparison to parents of normally developing children. Chronic stress could negatively influence parents' health while also impacting their ability to meet their child's needs. Despite this, there is a subset of parents who remain resilient in the face of significant stress in their lives. Knowledge of the factors that promote parental resilience could positively impact the services these families receive. The authors conducted a systematic review of research article databases and found support for coping style, optimism, and social support as resilience factors for parents of children with intellectual and/or developmental disabilities. Awareness of variables that promote resilience for parents of children with intellectual and/or development disabilities is likely to inform clinical practice through offering new avenues for clinical focus in all phases of family-centered care.
\end{abstract}

Keywords: developmental disabilities, intellectual disability, parenting, resilience, stress

\section{INTRODUCTION}

Parents of children with intellectual and/or developmental disabilities are presented with a unique set of challenges associated with providing care for their children. Depending upon the nature and severity of the disability, the child may require significant personal care and monitoring to ensure his or her wellness and safety. Parents are usually the primary provider of this care. Given other responsibilities that parents typically have in relation to maintaining stability for their family, the additional responsibility of providing significant levels of care for their child indefinitely may task parents' ability to maintain balance in their own lives. This may lead to stress for parents which could compromise their ability to effectively care for their child. Literature reveals that parents of children with intellectual and developmental disabilities do indeed experience significant levels of stress (Bouma \& Schweitzer, 1990; Cushner-Weinstein et al., 2008; Dumas, Wolf, Fisman, \& Culligan, 1991; Hussain \& Juyal, 2007; Kasari \& Sigman, 1997).

The stress experienced by parents not only negatively impacts their ability to care for their child, but also affects their

Received July 5, 2012; accepted September 14, 2013

Correspondence: Justin W. Peer, PhD, University of Michigan-Dearborn, 4012 CASL, 4901 Evergreen, Dearborn, MI 48128, USA.

Tel: +1 313593 5520; E-mail: jwpeer@umich.edu own mental and physical health, as well as the quality of the relationship with their child. Miodrag and Hodapp (2010) performed a comprehensive review of studies that analyzed the impact of chronic stress on health outcomes for parents of children with developmental disabilities and found that stress appears to have a negative influence on parent health, especially for mothers. The authors concluded that parenting stress could become a significant public health concern, as chronic health problems brought about by stress can negatively impact a mother's ability to provide necessary care for the child and disrupt the parent-child relationship. This suggestion is supported in a study by Mitchell and Hauser-Cram (2010) in which early childhood predictors of both positive and negative parent-adolescent relationships for parents with adolescents with developmental disabilities were investigated. Parenting stress early in a child's life was a significant variable in the quality of relationship, as higher stress levels when the child was young were significantly tied to poorer relationships between parent and the child later in life. These studies support that parents not only experience significant stress related to caring for their child with an intellectual and/or developmental disability, but this stress can also impact their ability to effectively parent their child, which could potentially have a long-term impact on the quality of the parent-child relationship. This stress could also have an adverse impact on parents' psychological well-being as evidenced by increased anxiety and depression (Cramm \& Nieboer, 2011). These findings provide insight into how stress permeates many 
important areas of functioning for parents and the deleterious effects that stress has on parental wellness.

McCubbin and Patterson (1983), as part of their Double ABCX Model of Family Stress, assert that difficulties that arise typically do so based upon the "pile-up" nature of stressors experienced. Specifically, parents likely experience stress due to the multiple aspects of maintaining family balance that they must contend with simultaneously. It is suggested that the overlapping nature of multiple stressors on a regular basis is what leads parents/families to feel taxed and, ultimately, in some way maladjusted.

Interestingly, research also reveals that despite the increased stress that parents experience, many still are able to manage successfully in the face of this adversity (Graungaard, Andersen, \& Skov, 2011; Green, 2007; Raina et al., 2005). A logical question that follows is "What is it that allows these parents to manage effectively despite the increased demands related to caring for their child with an intellectual and/or developmental disability?" An answer to this question may lie in the area of resilience, defined as the "ability to withstand and rebound from crisis and distress” (Heiman, 2002, p. 159).

\section{Resilience and Parenting Children With Intellectual and Developmental Disabilities}

Though broadly defined previously, resilience needs to be operationally defined and the construct further dissected for a thorough understanding of this concept to be obtained. Luthar and Cicchetti (2000) define resilience as a "process wherein individuals display positive adaptation despite experiences of significant adversity or trauma" (p. 858). The authors further identify resilience as a two-dimensional, dynamic construct wherein individuals have been exposed to both adversity and positive adaptation outcomes. Luthar and Cicchetti identify adversity, or risk, as being "negative life circumstances that are known to be statistically associated with adjustment difficulties" (p. 858) while positive adaption is noted as being "behaviorally manifested social competence or success at meeting stage-salient developmental tasks" (p.858). This is in contrast to competing definitions of resilience that have conceptualized resilience as a personal attribute/personality trait. The authors add that, depending upon the situation, absence of emotional and behavioral problems may be an appropriate indicator of adaptation.

This framework correlates highly with the experiences of a parent of a child with an intellectual and/or developmental disability. For these parents, there is risk associated with parenting their child. As cited in the previous section, empirical studies have tied parenting stress to poorer mental and physical health outcomes, among other adjustment-related difficulties. However, despite this, there are parents that experience "positive adaptation outcomes" despite the risk that exists.

Why does this occur? Luthar and Cicchetti (2000), within their resilience framework, suggest that there are both "vulnerability factors" and "protective factors" that either diminish or promote one's ability to be resilient. Vulnerability factors are identified as those variables that perpetuate the negative aspects of the risk situation while protective factors change the impact of the risk condition in a positive manner. The difference between the two is based upon the effect of the particular factor. If the lack of a factor in one's life promotes significant difficulties, then it is a vulnerability factor. If the presence of something in a person's life provides an advantage to an individual, then it is considered to be a protective factor (Luthar, 2006; Luthar \& Cicchetti, 2000).

As has been stated, there exists a cross-section of parents of children with disabilities that are able to be resilient despite the notable risks that are present. An examination of variables that may provide a protective psychological barrier for parents while preventing the negative outcomes associated with chronic stress from occurring is of the utmost importance for those providing professional services for these families.

\section{Aim of the Present Review}

What are the resilience factors associated with wellness for parents of children with intellectual and/or developmental disabilities? Gaining an understanding of what promotes resilience is of great significance for both parents and professionals. Parents can aid themselves through increased knowledge of what has helped others in maintaining balance despite the stressors surrounding them. For mental health professionals, family service workers, and others working with parents of children with intellectual and/or developmental disabilities, gaining an understanding of these variables can offer a significant tool when assessing or offering interventions for these families. This could provide clinicians and other family service workers a great opportunity to offer a proactive approach to working with these families, as education could be provided to parents on how to cultivate and hone resilience skills prior to the development of significant problems resulting from stress. For parents already experiencing significant stress, understanding key information regarding stress resilience can offer a foundation for an intervention designed to increase the family's functional level.

Providing families the necessary tools to maintain balance and resilience is critical for both parent and child wellness. If parents are experiencing significant stress, it is possible that they are struggling to provide adequate care for their child. This, in turn, negatively impacts the child's well-being and may interrupt movement toward reaching his/her developmental potential. A foundation of knowledge about factors that protect families from stress is key for parents and professionals alike. Given this, the aim of this article is to offer a comprehensive review of resilience factors for parents of children with intellectual and/or developmental disabilities. Another aim is to discuss the implications of these findings and offer recommendations for practitioners.

\section{METHOD}

A comprehensive review of three major online databases (CINAHL, PubMed, and PsycINFO) was utilized to gather relevant literature. Peer-reviewed quantitative and qualitative articles that were written in English and published between 1986 
and 2012 were initially included. Keywords including "parenting," "stress," "intellectual disabilities," "resilience," and "developmental disabilities" were used during the initial data base search to identify articles to include in the review. Articles retrieved were then reviewed and thematically organized. A secondary search adding the keywords "coping style," "optimism," "positive emotions," and "social support" was then conducted. These terms were utilized due to their high frequency of appearance within the initial search. A tertiary review was conducted after thoroughly reading the reference sections of secondary search articles. This produced further literature for use within this review.

\section{RESULTS}

\section{Coping Style as a Resilience Factor}

Stress, according to Lazarus as part of his Transactional Theory of Stress and Coping, results if an individual appraises a transaction with the environment as taxing and exceeding personal resources (Folkman \& Lazarus, 1986). It has been established that parents of children with intellectual and/or developmental disabilities experience significant stress in comparison to parents with children of typical development. Individuals must find a way to manage stress, which leads to a coping response. Lazarus refers to coping as a person's cognitive and behavioral efforts to manage stress-related demands resulting from environmental transactions that are perceived to be taxing or exceeding personal resources (Folkman, Lazarus, Dunkel-Schetter, DeLongis, \& Gruen, 1986). There are two distinct types of coping that are utilized in this process: (1) altering internal or external situational demands and problem solving to prevent the stressful situation from occurring in the future (problem-focused coping); and (2) providing regulation to the emotion caused by the situation (emotion-focused coping). An example of a problem-focused coping effort would be planful problem solving (deliberate analytic efforts to alter a situation and solve a problem) while escape avoidance (wishful thinking and behavioral efforts to prevent having to address the situation) is an emotion-focused coping strategy.

Is one form of coping more effective than the other? In terms of specific coping strategies, Folkman et al. (1986) argue that the coping process and strategies selected are not inherently good or bad. Rather, it is suggested that the adaptive qualities of the coping effort should be evaluated within the context of the specific situation in which it occurred. A particular coping process may be successful within one context and not in another. In review of the available literature, several studies have found problem-focused coping to be more effective than emotionfocused coping in providing long-term relief from stress. Miller, Gordon, Daniele, and Diller (1992), in a study of stress appraisal and coping style in mothers of children with disabilities, found that emotion-focused coping was significantly related to increased psychological distress whereas the use of problemfocused coping was tied to decreased distress. Smith, Seltzer, Tager-Flusberg, Greenberg, and Carter (2008) investigated the impact of coping style on maternal well-being of mothers of children with autism and found that increased use of problemfocused strategies was generally correlated with greater maternal well-being, regardless of the severity of the disorder.

Similarly, Glidden and Natcher (2009) investigated the relationship between coping strategies, personality, and adjustment for parents of children with developmental disabilities, and found that early use of problem-focused coping strategies by parents of children with developmental disabilities predicted less worry and greater subjective well-being. Lastly, Graungaard et al. (2011) found that parents of children with severe developmental disabilities were able to maintain a sense of personal well-being through the use of positive reappraisal, a problem-focused coping strategy.

Walsh's $(1996 ; 1998 ; 2003)$ family resilience framework offers further support for problem-focused coping strategies as resilience factors for parents. Within the family framework, key variables and processes are thought to aid families in building resilience. Walsh categorizes these processes into three primary categories: family belief systems, organization patterns, and communication processes. Under the family belief systems, category-specific processes include "making meaning of adversity" and "transcendence and spirituality." These strategies focus on reinterpreting crises as challenges and growing psychologically through adversity. Both of these processes correlate strongly with Lazarus' concept of "positive reappraisal" (Folkman et al., 1986) which Carver, Scheier, and Weintraub (1989) assert is strongly tied to problem-focused coping. Further, under the category of communication processes, Walsh states that "collaborative problem-solving," or the ability for families to proactively focus on goals and plan for the future, is a key resilience factor for families. The Transactional Model of Stress and Coping labels a very similar process as "planful problem-solving." As has been described, the family resilience framework offers strong theoretical support for coping serving as a buffer for parents related to the adversity they experience.

In summary, coping appears to effectively buffer parents from stress. In particular, utilizing a problem-focused coping style is related to lower stress levels for parents of children with intellectual and/or developmental disabilities. This style appears to protect parents from the potential emotional upheaval associated with their role and promotes a sense of resilience in their lives. As parents of children with disabilities are confronted with many situations that strain their personal resources to overcome obstacles, understanding that there are particular ways that parents can remain resilient, or build resilience, while navigating these issues provides hope and promise for all involved.

\section{Optimism as a Resilience Factor}

Dispositional optimism, or the tendency to expect positive outcomes when confronting problems in life (Scheier et al., 1989), appears to be a key factor in protecting individuals from stress. The field of positive psychology, led by Martin Seligman, suggests that optimism is a factor that promotes a high quality of life while also preventing psychopathology and emptiness (Seligman \& Csikszentmihalyi, 2000). Positive psychology theorists believe that a tendency toward optimism for the future is a valued trait that has a significant impact on the individual. 
People high in optimism are hypothesized to have better moods, are able to generate positive emotion more readily, are more persevering and successful, are more effective in problem solving, and experience better physical health according to Seligman and Csikszentmihalyi (2000). Folkman (2008), in a comprehensive review of positive outlook and emotion in relation to stress, states that positive emotions are a natural part of the stress process and play an important role in re-establishing physiological and psychological resources. This is an important variable in the pursuit of resilience for parents of children with intellectual and/or developmental disabilities. If parents can maintain, or improve upon, a positive attitude and hope for the future despite the stressors surrounding them, this could have a significant impact on parents' ability to maintain the provision of both adequate child care and self-care.

Research supports dispositional optimism as a resilience factor for parents of children with disabilities. Kayfitz, Gragg, and Orr (2010) explored the impact that positive experiences had on mothers and fathers of children with autism, and found that positive experience scores were significantly negatively correlated with parental distress. This suggests that parents who were able to view the contributions of their child more positively experienced relief from the parenting stress associated with caring for their child. The authors speculate that having a more positive approach to viewing their child allows parents to pay less attention to the child's limitations and, in turn, potentially pay less attention to their limitations as parents, which offers protection against a negative sense of well-being.

Baker, Blacher, and Olsson (2005) conducted a study investigating the impact of optimism on measures of well-being for parents of preschool children with and without developmental delays. The study primarily explored the correlation between child behavior problems and various measures of parental wellbeing (e.g., depression, marital adjustment), as well as the moderating impact of optimism. Optimism was found to moderate the relationship between behavior problems and parental wellbeing, especially for mothers. Mothers who were rated as being less optimistic reported lower scores on measures of well-being when child behaviors were high, more so than mothers who were more optimistic.

Similarly, in a study conducted by Karazsia and Wildman (2009), the mediating role of parenting behaviors on positive affect and negative affect in the context of child behavior problems was explored. The authors hypothesized that parental self-reports of positive affect (the extent to which a person experiences positive thoughts and emotions) would be related to child behavior problems. Results indicated that increased levels of parental positive affect were associated with decreased problem behaviors by the child. Also, high levels of positive affect were significantly correlated with low levels of maladaptive parenting behaviors. Based on this study, it is reasonable to assert that fewer child difficulties and more adaptive parenting produce less stress for parents, thus reinforcing the importance of positive emotions in relation to stress management/resilience.

In related studies investigating the contribution of optimism to stress and well-being, Aspinwall and Grunhart (2000) found that higher levels of optimism facilitated the ability to process information related to health and that optimistic beliefs may play an especially important and beneficial role in earlier stages of the coping process. The authors speculate that the traits of optimism, in conjunction with coping, may offer some benefits in managing multiple life stressors. Also, de Schipper, Riksen-Walraven, Geurts, and Derksen (2008) conducted a study comparing carer mood and quality of interaction between carers and the children in their care, and found that optimism contributed significantly to both the quality of carers behavior toward children and to the children's well-being. Walsh (2003) lends support for optimism serving as a resilience factor for parents within her "family resilience framework." Walsh identifies having a "positive outlook" as a key resilience process for families. Positive outlook is conceptualized as having a sense of hope for the future as well as possessing an "optimistic bias" related to challenges in one's life. It is stated that this is a vital component for high-functioning families.

Though this characteristic may superficially appear similar to coping, having a positive outlook clearly varies from coping in both breadth and depth. Whereas coping is a response to stress, optimism is a disposition that influences an individual's effort to persevere even when situations are difficult (Scheier et al., 1989). Having a positive view appears to allow parents to maintain and restore resources necessary to effectively manage the stress associated with caring for their child. This may occur through a parent's ability to look past, and not dwell on, any negative aspects of parenting their child while focusing on the strengths their child possesses and the happiness that being a parent brings each and every day. These parents may also be more hopeful about the future, which generates a sense of well-being. This aids in understanding how parents are able to manage effectively despite the stressors present in their lives. The aforementioned findings support the importance of positive disposition/optimism as a prime resilience factor for parents while also demonstrating the uniqueness of this quality.

\section{Social Support as a Resilience Factor}

From this review of the current literature, the availability of family, friends, and others to offer support to parents of children with intellectual/developmental disabilities appears to be a particularly strong predictor of resilience. Research holds that having both formal and informal supports available for assistance and consultation buffers parents from the negative effects of stress.

In a study of social support, Langford, Bowsher, Maloney, and Lillis (1997) found that social support was linked to various positive health states that included effective coping behaviors, sense of stability, psychological well-being, and perceived control among others. This linkage to the outside world to receive regard and assistance appears to negate the impact that stress can have on parental well-being. Other research supports this position. Sipal, Schuengel, Voorman, Van Eck, and Becher (2010) examined the impact that parenting stress and social support had on the course of behavioral problems for children with cerebral palsy. Their study's primary aim was to test whether parenting stress and social support played a significant role in the course of behavior problems for the child. Findings revealed that lack of environmental support significantly correlated with not only stress experienced by parents in their relationships with their child, but also stress associated with the marital relationship. 
Pottie and Ingram (2008) conducted a study investigating daily stress, coping, and well-being in parents of children with autism. Specifically, the moderating effects of coping on the distress experienced by parents as well as the overall well-being of parents were examined. Social support was found to moderate the daily stress/mood relationship. The authors suggest that social support can potentially enhance well-being and reduce daily distress in parents of children with autism.

Beckman (1991), in a study comparing the perceptions of parents of children with and without disabilities, found that stress was negatively correlated with informal support (friends, family). Similarly, Spratt, Saylor, and Macias (2007) conducted a study that also investigated correlates of stress for parents of children with developmental disabilities. However, in this study, a broader range of disabilities were analyzed. The sample included children experiencing a variety of health, developmental, behavioral, and neurological problems. Utilizing a multiple regression analysis, the authors found that the perceived inadequacy of family support and maternal support were related to parenting stress in the samples investigated. Further, Smith, Oliver, and Innocenti (2001) found in their study of parenting stress in families of children with disabilities that the variable of social support predicted parenting stress more accurately than variables related to the child's level of functioning. Lastly, Peer and Hillman (2012) found social support to be a significant predictor of stress for parents of individuals with intellectual disabilities.

Related to formal social supports, Heiman (2002), in a study exploring the role of social support as a resilience factor for parents, found that having the ability to openly discuss concerns with both family and professionals appears to promote resilience for families of children with disabilities. Also, Freedman, Litchfield, and Warfield (1995) found formal social support to be a key stress management factor for working parents of children with developmental disabilities. Also, Cowen and Reed (2002) examined the impact of respite care received by at-risk families of children with developmental disabilities on stress levels. A comparison of scores on stress measures given before and after formal respite services were rendered, revealed significant decreases in total stress for the parents in the study.

Social support appears to be uniquely tied to resilience for parents of children with disabilities. The evidence is clear in describing the relationship between social support and stress management. Developing and maintaining a strong social network appear to be of primary importance for families of children with intellectual and/or developmental disabilities to maintain cohesiveness and stability in the midst of the stress that coincides with responsibilities placed upon these parents. Having others available seems to help alleviate both the physical and emotional burden that parents may feel related to their responsibilities. Also, having a stable social network likely provides a sounding board for parents, allowing them to ventilate pent-up negative feelings that they may be experiencing related to the demands being placed upon them. It is critical that parents have a stable informal (family, friends) or formal (professionals, support groups) group to provide support and reassurance for them as they provide care for their child.

\section{DISCUSSION}

The results of this review suggest that coping style, optimism, and social support are all factors that may influence resilience for parents of children with developmental disabilities. These findings are likely to inform clinical practice through advancements in assessment, planning, and intervention.

\section{Implications and Recommendations}

Parents of children with intellectual and/or developmental disabilities as a group experience significant levels of stress (Bouma \& Schweitzer, 1990; Cushner-Weinstein et al., 2008; Dumas et al., 1991; Hussain \& Juyal, 2007; Kasari \& Sigman, 1997). An exploration of parental coping, optimism, and social support offers practitioners insight into factors that potentially mediate the effects of stress for parents, allowing them to preserve personal resources so that they can effectively care for their children. This knowledge offers practitioners from various disciplines (e.g., psychologists, social workers, family service workers, etc.) an excellent opportunity to be proactive in their interactions with these families to promote wellness and, ultimately, resilience. Given the importance of stress related to the process of parenting children with disabilities, the findings discussed should influence both assessment and intervention for practitioners.

Related to assessment, identifying where parents lie along the continuum of coping, optimism, and social support should be a critical evaluation component for professionals. Assessing these areas will provide clinicians with valuable data related to both the level and etiology of stress that the family may be experiencing. It is recommended that practitioners ask basic questions regarding how parents generally cope with life demands, their level of optimism about the present and future, as well as the nature and stability of their social support network. Practitioners are encouraged to explore assessment tools available to evaluate these areas. Assessment questionnaires exist that are both brief and psychometrically sound to rate these areas. Examples include the brief COPE (Carver, 1997) to identify coping strategies, the Multidimensional Scale of Perceived Social Support (Zimet, Dahlem, Zimet, \& Farley, 1988) to measure social support, and the Revised Life Orientation Test (Scheier, Carver, \& Bridges, 1994) to assess optimism. Having this data will not only provide the clinician with an important understanding about the potential roots of parental stress, but should also influence the design and implementation of interventions for parents that will promote the cultivation of resilience skills.

As for interventions, professionals can utilize the information gained via the assessment to proactively address any noted concerns, or to continue to build resilience already present. It is recommended that professionals provide skill training to parents regarding these areas along with educational materials or brochures to facilitate learning. If the practitioner is trained and able to provide psychotherapy, supportive counseling is recommended to address any deficits in effective coping and to restructure negative/pessimistic thought processes with more positive/ 
adaptive ones. If the professional is not trained to do so, referral for psychotherapy would be appropriate.

Regarding social supports, the practitioner is a vital component in providing such supports for families and should embrace the importance of this role. Professionals should also make referrals to any available and appropriate support groups to expand the social support network for the parents they serve. Clinicians should also work directly with parents to explore ways that natural supports could be integrated into daily routines in an effort to alleviate stress while also educating families regarding the importance of family and friends as instrumental and emotional supports.

It is further recommended that practitioners offer these supports in an environment where family strengths are acknowledged and a sense of hope is communicated (Bayat, 2007; Bayat \& Schuntermann, 2013). This may provide families a positive outlook for the future, thus facilitating the development of resilience. Additionally, Shapiro (2002) noted that professionals should focus on the family's own interpretation of their current life circumstances while also normalizing their experiences and the attempts they are making to manage adversity.

Professionals play an essential role in helping families successfully navigate the challenges associated with caring for their child with an intellectual and/or developmental disability. Utilizing these findings can aid practitioners in being more effective in their role while simultaneously helping parents be more effective in theirs. Proactively approaching stress in this manner may offer families greater peace and a sense of confidence in their ability while allowing the professional the opportunity to focus on other important need areas for the family. This is an effective way to help parents maintain or develop a sense of resilience which the research suggests is vital for stress management.

\section{Directions for Future Research}

Based on the findings, it is suggested that future studies empirically measure the relationship between various aspects of the factors discussed here and stress among parents of children with disabilities. Further, research should explore other variables that may promote resilience for parents. Lastly, it is suggested that studies seek to validate practice methods that may facilitate the development or maintenance of resilience for parents.

\section{CONCLUSION}

Research reveals that parents of children with intellectual and/or developmental disabilities experience significant levels of stress. Despite this, some parents are still able to manage effectively and provide effective care for their child. Coping style, optimism, and social support have all been found to be strong predictors of resilience for parents of children with disabilities. This provides practitioners with a new and proactive avenue for both assessment and treatment of parental stress. This is critical, considering how important preserving personal resources are for these parents as they navigate child-care needs and daily hassles simultaneously. Understanding the factors that build resilience within parents informs clinical practice and increases a professional's ability to be an influential partner in helping parents of children with intellectual and/or developmental disabilities be effective carers.

\section{REFERENCES}

Aspinwall, L. G., \& Grunhart, S. M. (2000). What I do know won't hurt me: Optimism, attention to negative information, coping, and health. In J. E. Gillham (Ed.), The science of optimism and hope symposium (pp. 163-200), West Conshohocken, PA: Templeton Foundation Press.

Baker, B. L., Blacher, J., \& Olsson, M. B. (2005). Preschool individuals with and without developmental delay: Behaviour problems, parents' optimism and well-being. Journal of Intellectual Disability Research, 49, 575-590.

Bayat, M., \& Schuntermann, P. (2013). Enhancing resilience in families of children with autism spectrum disorder. In D. S. Becvar (Ed.), Handbook of family resilience (pp. 409-424). New York, NY: Springer Science + Business Media.

Bayat, M. M. (2007). Evidence of resilience in families of children with autism. Journal of Intellectual Disability Research, 51, 702-714.

Beckman, P. J. (1991). Comparison of mothers' and fathers' perceptions of the effect of young individuals with and without disabilities. American Journal on Mental Retardation, 95, 585-585.

Bouma, R., \& Schweitzer, R. (1990). The impact of chronic childhood illness on family stress: A comparison between autism and cystic fibrosis. Journal of Clinical Psychology, 46, 722-730.

Carver, C. S. (1997). You want to measure coping but your protocol's too long: Consider the brief COPE. International Journal of Behavioral Medicine, 4, 92-100.

Carver, C. S., Scheier, M. F., \& Weintraub, J. K. (1989). Assessing coping strategies: A theoretically based approach. Journal of Personality and Social Psychology, 56, 267-283.

Cowen, P. S., \& Reed, D. A. (2002). Effects of respite care for children with developmental disabilities: Evaluation of an intervention for at risk families. Public Health Nursing, 19, 272-272.

Cramm, J. M., \& Nieboer, A. P. (2011). Psychological well-being of caregivers of children with intellectual disabilities: Using parental stress as a mediating factor. Journal of Intellectual Disabilities, 15, 101-113.

Cushner-Weinstein, S., Dassoulas, K., Salpekar, J. A., Henderson, S. E., Pearl, P. L., Gaillard, W. D., \& Weinstein, S. L. (2008). Parenting stress and childhood epilepsy: The impact of depression, learning, and seizure-related factors. Epilepsy \& Behavior, 13, 109-114.

de Schipper, E. J., Riksen-Walraven, J. M., Geurts, S. A. E., \& Derksen, J. J. L. (2008). General mood of professional caregivers in child care centers and the quality of caregiver-child interactions. Journal of Research in Personality, 42, 515-526.

Dumas, J. E., Wolf, L. C., Fisman, S. N., \& Culligan, A. (1991). Parenting stress, child behavior problems, and dysphonia in parents of individuals with autism, Down syndrome, behavior disorders, and normal development. Exceptionality, 2, 97-110.

Folkman, S. (2008). The case for positive emotions in the stress process. Anxiety, Stress \& Coping, 21, 3-14.

Folkman, S., \& Lazarus, R. S. (1986). Stress processes and depressive symptomatology. Journal of Abnormal Psychology, 95, 107-113.

Folkman, S., Lazarus, R. S., Dunkel-Schetter, C., DeLongis, A., \& Gruen, R. J. (1986). Dynamics of a stressful encounter: Cognitive appraisal, coping, and encounter outcomes. Journal of Personality and Social Psychology, 50, 992-1003.

Freedman, R. I., Litchfield, L. C., \& Warfield, M. (1995). Balancing work and family: Perspectives of parents of children with developmental disabilities. Families in Society, 76, 507-514. 
Glidden, L. M., \& Natcher, A. L. (2009). Coping strategy use, personality, and adjustment of parents rearing children with developmental disabilities. Journal of Intellectual Disability Research, 53, 998-1013.

Graungaard, A., Andersen, J., \& Skov, L. (2011). When resources get sparse: A longitudinal, qualitative study of emotions, coping and resource-creation when parenting a young child with severe disabilities. Health: An Interdisciplinary Journal for the Social Study of Health, Illness and Medicine, 15, 115-136.

Green, S. E. (2007). "We're tired, not sad": Benefits and burdens of mothering a child with a disability. Social Science and Medicine, 64, $150-163$.

Heiman, T. (2002). Parents of children with disabilities: Resilience, coping, and future expectations. Journal of Developmental and Physical Disabilities, 14, 159-171.

Hussain, A., \& Juyal, I. (2007). Stress appraisal and coping strategies among parents of physically challenged individuals. Journal of the Indian Academy of Applied Psychology, 33, 179-182.

Karazsia, B. T., \& Wildman, B. G. (2009). The mediating effects of parenting behaviors on maternal affect and reports of children's behavior. Journal of Child and Family Studies, 18, 342-349.

Kasari, C., \& Sigman, M. (1997). Linking parental perceptions to interactions in young individuals with autism. Journal of Autism and Developmental Disorders, 27, 39-57.

Kayfitz, A. D., Gragg, M. N., \& Orr, R. R. (2010). Positive experiences of mothers and fathers of individuals with autism. Journal of Applied Research in Intellectual Disabilities, 23, 337-343.

Langford, C. P. H., Bowsher, J., Maloney, J., \& Lillis, P. P. (1997). Social support: A conceptual analysis. Journal of Advanced Nursing, 25, 95-100.

Luthar, S. S. (2006). Resilience in development: A synthesis of research across five decades. In D. Cicchetti \& D. J. Cohen (Eds.), Developmental psychopathology: Risk, disorder, and adaptation (pp. 740795). New York, NY: Wiley.

Luthar, S. S., \& Cicchetti, D. (2000). The construct of resilience: Implications for interventions and social policy. Development and Psychopathology, 12, 857-885.

McCubbin, H. I., \& Patterson, J. M. (1983). The family stress process: The double ABCX model of adjustment and adaptation. Marriage \& Family Review, 6, 7-37.

Miller, A. C., Gordon, R. M., Daniele, R. J., \& Diller, L. (1992). Stress, appraisal, and coping in mothers of disabled and nondisabled individuals. Journal of Pediatric Psychology Special Issue: Theory-Driven Research in Pediatric Psychology: I, 17, 587-605.

Miodrag, N., \& Hodapp, R. M. (2010). Chronic stress and health among parents of children with intellectual and developmental disabilities. Current Opinion in Psychiatry, 23, 407-411.

Mitchell, D. B., \& Hauser-Cram, P. (2010). Early childhood predictors of mothers' and fathers' relationships with adolescents with developmental disabilities. Journal of Intellectual Disability Research, 54, 487-500.

Peer, J. W., \& Hillman, S. B. (2012). The mediating impact of coping style on stress perception for parents of individuals with intellectual disabilities. Journal of Intellectual Disabilities, 16, 45-59.

Pottie, C. G., \& Ingram, K. M. (2008). Daily stress, coping, and wellbeing in parents of individuals with autism: A multilevel modeling approach. Journal of Family Psychology, 22, 855-864.

Raina, P., O’Donnell, M., Rosenbaum, P., Brehaut, J., Walter, S. D., Russell, D., ... Wood, E. (2005). The health and well-being of caregivers of children with cerebral palsy. Pediatrics, 115, 626-636.

Scheier, M. F., Carver, C. S., \& Bridges, M. W. (1994). Distinguishing optimism from neuroticism (and trait anxiety, self-mastery, and self-esteem): A reevaluation of the life orientation test. Journal of Personality and Social Psychology, 67, 1063-1078.

Scheier, M. F., Matthews, K. A., Owens, J. F., Magovern, G. J., Lefebvre, R., Abbott, R., \& Carver, C. S. (1989). Dispositional optimism and recovery from coronary artery bypass surgery: The beneficial effects on physical and psychological well-being. Journal of Personality and Social Psychology, 57, 1024-1040.

Seligman, M. E. P., \& Csikszentmihalyi, M. (2000). Positive psychology: An introduction. American Psychologist, 55, 5-14.

Shapiro, E. R. (2002). Chronic illness as a family process: A socialdevelopmental approach to promoting resilience. Journal of Clinical Psychology, 58, 1375-1384.

Sipal, R. F., Schuengel, C., Voorman, J. M., Van Eck, M., \& Becher, J. G. (2010). Course of behaviour problems of children with cerebral palsy: The role of parental stress and support. Child Care, Health \& Development, 36, 74-84.

Smith, L. E., Seltzer, M. M., Tager-Flusberg, H., Greenberg, J. S., \& Carter, A. S. (2008). A comparative analysis of well-being and coping among mothers of toddlers and mothers of adolescents with ASD. Journal of Autism and Developmental Disorders, 38, 876-889.

Smith, T. B., Oliver, M. N. I., \& Innocenti, M. S. (2001). Parenting stress in families of individuals with disabilities. American Journal of Orthopsychiatry, 71, 257-257.

Spratt, E. G., Saylor, C. F., \& Macias, M. M. (2007). Assessing parenting stress in multiple samples of children with special needs (CSN). Families, Systems, \& Health, 25, 435-449.

Walsh, F. (1996). The concept of family resilience: Crisis and challenge. Family Process, 35, 261-281.

Walsh, F. (1998). Strengthening family resilience. New York, NY: Guilford Press.

Walsh, F. (2003). Family resilience: A framework for clinical practice. Family Process, 42, 1-18.

Zimet, G. D., Dahlem, N. W., Zimet, S. G., \& Farley, G. K. (1988). The multidimensional scale of perceived social support. Journal of Personality Assessment, 52, 30-41. 